\title{
Structural Stability of the Generalized Taylor Rule
}

\author{
William A. Barnett* Evgeniya A. Duzhak ${ }^{\dagger}$
}

January 15, 2013

\begin{abstract}
This paper analyzes the dynamical properties of monetary models with regime switching. We start with the analysis of the evolution of inflation when policy is guided by a simple monetary rule where coefficients switch with the policy regime. We rule out the possibility of a Hopf bifurcation and demonstrate the existence of a period doubling bifurcation. As a result, a small change in the parameters (e.g. a more active policy response) can lead to a drastic change in the path of inflation. We demonstrate that while the New Keynesian model with a current-looking Taylor rule is not prone to bifurcations, a hybrid rule exhibits the same pattern of period doubling bifurcations as the basic setup.
\end{abstract}

JEL classification: C14, C22, E37, E32

Keywords: New Keynesian, Taylor Rule, regime switching, bifurcation analysis, structural stability.

*University of Kansas, 356 Snow Hall, Lawrence, KS 66045, USA; E-mail: barnett@ku.edu

${ }^{\dagger}$ Baruch College, City University of New York, Zicklin School of Business; Postal Address: 17 Lexington Avenue, New York, NY 10010, USA; E-mail: evgeniya_duzhak@baruch.cuny.edu. 


\section{Introduction}

Monetary policy has seen drastic changes over the past decades. The 1970s were plagued by high inflation along with slow economic growth while the Central bank stayed relatively passive in their actions. With the appointment of Volcker, the Federal Reserve shifted to a more active regime which helped to combat high inflation rates present at the start of the 1980s. The following period of moderate inflation along with stable economic growth has been coined the Great Moderation. With the Great Recession as a result of the financial crises starting in 2007, the Fed had to move aggressively.

This paper studies the dynamical behavior of standard macroeconomic models where the monetary policy regime can switch over time. More specifically, the policy regime follows a Markov chain with a fixed transition matrix. We are interested in the qualitative behavior of the solution not only for one particular calibration of the model but rather the change in the qualitative behavior of the solution in response to changes in the parameterization.

The standard New Keynesian model is known to display bifurcations, i.e. changes in the dynamical evolution of the variables of interest in response to changes in parameters (see Barnett and Duzhak (2008) and Barnett and Duzhak (2010)). The present paper investigates whether bifurcations are possible when regimes can switch over time. The answer is a-priori not clear. The solution to the model, in the simplest case inflation, evolves differently depending on the state the regime is in. If the standard New Keynesian model displays a bifurcation

for a certain region of the parameter space, then the regime switching model would visit this parameter combination only for one of the policy regimes while 
the occasional switch to another policy regime can stabilize the solution.

In the first section, we study a basic setup with exogenous real interest rates. The monetary authority sets the nominal interest rate as a function of current inflation. However, the response coefficient varies depending on the policy regime present at the time. The Fisher equation which links the nominal interest rate to future inflation and the real interest rate provides the second relationship. Combining the two, we get an equation that relates future inflation to current inflation and the real interest rate. Taking the latter as given, we get a system of two linear difference equations for inflation in the two regimes.

To perform bifurcation analysis, we study the matrix that governs the evolution of current inflation to future inflation. The relevant properties of this matrix are the sign and magnitude of the eigenvalues. Therefore, we set up and solve the characteristic polynomial.

The solution demonstrates two main findings with respect to bifurcations. First, for the basic setup, there is no possibility of a Hopf bifurcation which would lead to a change from a stable to unstable solution (or vice versa) in response to a (potentially tiny) change in the parameter values. Second, we show the existence of a period doubling bifurcation. In this case, the solution can move from a stable to a periodic solution where the periodicity doubles.

Next, we ask whether our analysis of this simple setup carries over to the standard New Keynesian model. There, the monetary policy rule is more involved. The so-called Taylor rule (see Taylor (1999)) has two components which makes the nominal interest rate a function of both inflation and the output gap. This extra component can lead the solution to become less prone to changes in its 
characteristics.

Due to the increased dimensionality for the New Keynesian economy as opposed ot the basic setup, we move to numerical methods. There, we start with a single bifurcation point and trace out the entire bifurcation boundary through the parameter space, i.e. the parameter combinations at which a bifurcation occurs.

We find that the standard New Keynesian model with regime switching and a standard Taylor rule does not exhibit any bifurcations for the range of feasible parameter combinations. While we do find a bifurcation boundary, it lies outside the relevant range of parameter values and points to negative coefficients where standard economics tells us that they should be positive.

Lastly, we investigate whether a state-of-the-art hybrid Taylor rule exhibits any bifurcations. We solve the same baseline New Keynesian model but use a Taylor rule that allows for forward looking response to inflation. After going through the same solution steps as in the previous case, we find that this model might exhibit a period-doubling bifurcation. The ideas from the basic setup thus carry over to the most prominent model of monetary policy.

Our paper relates to several strands of the literature. First, we use the New Keynesian model with regime switching and study its properties. The original New Keynesian model has been developed into an important tool for monetary policy (see Gali and Gertler (1999), Bernanke, Laubach, Mishkin and Posen (1999), and Leeper and Sims (1994)). Andrews (1993) and Evans (1985) study monetary policy with parameter instability. Davig and Leeper (2006) and Farmer, Waggoner and Zha (2007) study determinacy when the Taylor rule is generalized 
to allow for regime switching. There is a literature on methods to determine parameter instability in time series (see Hansen (1992) and Nyblom (1989)). Economic models of regime switching had then been investigated previously in different contexts, see e.g. Hamilton (1989) and Warne (2000). Clarida, Gali and Gertler (1999), Sims and Zha (2006) and Groen and Mumtaz (2008) find empirical support for regime switches in monetary policy.

Second, we relate and make use of a large literature on bifurcation analysis. Seydel (1994) provides an overview on theoretical insights and applications with respect to bifurcations. Benhabib and Nishimura (1979) is an early example of the use of bifurcations in economics. More recently, Barnett and He study various forms of economic settings (see Barnett and He (1999), Barnett and He (2001), Barnett and He (2002), Barnett and He (2004), and Barnett and He (2006)). The present authors investigate bifurcation properties of New Keynesian models (see Barnett and Duzhak (2008) and Barnett and Duzhak $(2010))$.

The rest of the paper is organized as follows. Section 2 discusses a simple version with exogenous real interest rates where nominal inflation is determined by a policy rule. Section 3 discusses the classic New Keynesian model with regime switching and a current-looking Taylor rule. Section 4 studies the same New Keynesian model with a hybrid Taylor rule where we pay attention to the forward looking aspect of policy. Section 5 concludes. 


\section{Dynamics with a simple monetary policy rule}

Before studying a complete New Keynesian macroeconomic model, we study the dynamical properties of the monetary policy rule. A Central banker is implementing a policy where he reacts to inflation by changing an interest rate according to:

$$
i_{t}=\alpha\left(s_{t}\right) \pi_{t},
$$

where $i_{t}$ is the nominal interest rate, $\alpha\left(s_{t}\right)$ a state-dependent coefficient which changes with the policy regime $s_{t}$, and $\pi_{t}$ denotes the rate of inflation.

We assume that there are two possible realizations for the policy regime $s_{t}$. The policy regime determines the reaction to inflation when setting the nominal interest rate. This linear reaction function to inflation evolves stochastically between two states $-s_{t}=1$ and $s_{t}=2$, so that

$$
\alpha\left(s_{t}\right)= \begin{cases}\alpha_{1} & \text { for } s_{t}=1 \\ \alpha_{2} & \text { for } s_{t}=2\end{cases}
$$

where $\alpha_{i}$ denotes different parameters that govern the aggressiveness of policy to combat inflation. As usual, an active policy regime is the one when policy parameter $\alpha_{i}>1$.

The policy regime evolves according to a Markov chain where the transitional probabilities are given by the transition matrix with entries $p_{i j}=P\left[s_{t}=\right.$ $\left.j \mid s_{t-1}=i\right]$ where $i, j=1,2$. Following Davig and Leeper (2006), we study 
the dynamics of this simple monetary policy rule by using the Fisher equation

$$
i_{t}=E_{t} \pi_{t+1}+r_{t}
$$

, where $r_{t}$ is the real interest rate.

The Fisher equation links the nominal interest rate to expected inflation and the real interest rate. We use this relationship to solve for expected inflation which evolves as a function of the nominal and real interest rates. Plugging in for the policy rule where the nominal interest rate is a function of inflation, we end up with the following dynamic system

$$
\left[\begin{array}{c}
E_{t}\left[\pi_{1 t+1}\right] \\
E_{t}\left[\pi_{2 t+1}\right]
\end{array}\right]=\left[\begin{array}{ll}
p_{11} & p_{12} \\
p_{21} & p_{22}
\end{array}\right]^{-1}\left[\begin{array}{cc}
\alpha_{1} & 0 \\
0 & \alpha_{2}
\end{array}\right]\left[\begin{array}{l}
\pi_{1 t} \\
\pi_{2 t}
\end{array}\right]-\left[\begin{array}{ll}
p_{11} & p_{12} \\
p_{21} & p_{22}
\end{array}\right]^{-1}\left[\begin{array}{l}
r_{t} \\
r_{t}
\end{array}\right]
$$

In this system, we take the real interest rate $r_{t}$ as exogenously given. A fully specified macroeconomic model, which we study below, endogenizes this rate.

We now analyze this system of linear difference equations. Therefore, we replace the matrix multiplying the vector of inflation by explicitly computing the inverse of the transition matrix

$$
\left[\begin{array}{ll}
p_{11} & p_{12} \\
p_{21} & p_{22}
\end{array}\right]^{-1}\left[\begin{array}{cc}
\alpha_{1} & 0 \\
0 & \alpha_{2}
\end{array}\right]=\frac{1}{\Delta}\left[\begin{array}{cc}
p_{22} & -p_{12} \\
-p_{21} & p_{11}
\end{array}\right]\left[\begin{array}{cc}
\alpha_{1} & 0 \\
0 & \alpha_{2}
\end{array}\right]=\left[\begin{array}{cc}
\frac{p_{22} \alpha}{\Delta} & \frac{-p_{12} \alpha_{2}}{\Delta} \\
\frac{-p_{21} \alpha_{1}}{\Delta} & \frac{p_{11} \alpha_{2}}{\Delta}
\end{array}\right]
$$

where $\Delta$ denotes the determinant of the transition matrix.

As is standard in the (bifurcation) analysis of difference equations, we study the economy with parameter certainty. Parameter certainty means that agents 
have no uncertainty about the level of inflation if a certain state occurs. This does not mean that agents know the level of inflation in the following period: The state of the policy regime determines inflation and the state of the policy regime itself switches with given probabilities. Using parameter certainty, we can replace the expected level of inflation conditional on a state occuring by the level of inflation.

Putting parameter certainty and the adjustments to the matrix and its determinant into our equation, we can restate the system of linear difference equations as

$$
\left[\begin{array}{l}
\pi_{1 t+1} \\
\pi_{2 t+1}
\end{array}\right]=\left[\begin{array}{ll}
\frac{p_{22} \alpha}{p_{11} p_{22}-p_{12} p_{21}} & \frac{-p_{12} \alpha_{2}}{p_{11} p_{22}-p_{12} p_{21}} \\
\frac{-p_{21} \alpha_{1}}{p_{11} p_{22}-p_{12} p_{21}} & \frac{p_{11} \alpha_{2}}{p_{11} p_{22}-p_{12} p_{21}}
\end{array}\right]\left[\begin{array}{l}
\pi_{1 t} \\
\pi_{2 t}
\end{array}\right]-\left[\begin{array}{ll}
p_{11} & p_{12} \\
p_{21} & p_{22}
\end{array}\right]^{-1}\left[\begin{array}{l}
r_{t} \\
r_{t}
\end{array}\right]
$$

Since the entries in the transition matrix are probabilities, we know that $p_{11}+$ $p_{21}=1$ and $p_{22}+p_{12}=1$. Hence, we can express $\Delta=p_{11} p_{22}-p_{12} p_{21}$ as $\Delta=p_{11}+p_{22}-1$.

The key component of the dynamical system is the coefficient on current inflation. This Jacobian of the linear difference equation captures the evolution of expected inflation and thus the link between real and nominal interest rates. Given our calculations for the matrix on inflation and the determinant of the transition matrix above, the Jacobian of the linear difference equation is given by

$$
J=\left[\begin{array}{cc}
\frac{p_{22} \alpha_{1}}{p_{11}+p_{22}-1} & \frac{-p_{12} \alpha_{2}}{p_{11}+p_{22}-1} \\
\frac{-p_{21} \alpha_{1}}{p_{11}+p_{22}-1} & \frac{p_{11} \alpha_{2}}{p_{11}+p_{22}-1}
\end{array}\right]
$$


To analyze the stability of the evolution of inflation and its dynamic properties, we compute the eigenvalues for the Jacobian matrix. Therefore, we compute the characteristic polynomial $P(\lambda)$ which is quadratic in this case given by

$$
P(\lambda)=\lambda^{2}-b \lambda+c
$$

where the coefficients are

$$
b=\frac{p_{22} \alpha_{1}+p_{11} \alpha_{2}}{p_{11}+p_{22}-1} \text { and } c=\frac{\alpha_{1} \alpha_{2}}{\left(p_{11}+p_{22}-1\right)}
$$

The nature of the solution to quadratic equations is mainly determined by the discriminant of the square root that appears in the formula. For the dynamics of inflation, the determinant $D$ is given by

$$
D=\left[\frac{p_{22} \alpha_{1}+p_{11} \alpha_{2}}{p_{11}+p_{22}-1}\right]^{2}-\frac{4 \alpha_{1} \alpha_{2}}{\left(p_{11}+p_{22}-1\right)}
$$

A negative discriminant $D$ gives rise to complex roots whereas a positive discriminant leads to real roots.

We are interested in the quality of the dynamics and whether bifurcation exists, i.e. whether the quality of the solution can change drastically despite a negligible change in the parameter values. For a Hopf bifurcation (Hopf (1942)) to exist, the discriminant $D$ must be negative, giving a rise to complex roots of the characteristic polynomial. To check whether this is true, we need to solve for

$$
D=\frac{\left(p_{22} \alpha_{1}+p_{11} \alpha_{2}\right)^{2}}{\left(p_{11}+p_{22}-1\right)^{2}}-\frac{\left(p_{11}+p_{22}-1\right) 4 \alpha_{1} \alpha_{2}}{\left(p_{11}+p_{22}-1\right)^{2}}<0
$$


Given that $\left(p_{11}+p_{22}-1\right)^{2}$ is always nonnegative, we can simplify the inequality to

$$
\left(p_{22} \alpha_{1}+p_{11} \alpha_{2}\right)^{2}-\left(p_{11}+p_{22}-1\right) 4 \alpha_{1} \alpha_{2}<0 .
$$

The term on the left-hand side stays positive within the feasible set of parameters. Therefore, a Hopf bifurcation which arises only when the roots are complex, is not possible for this economy.

However we can check the possibility of a Period Doubling bifurcation. This type of bifurcation occurs when the root equals negative one and it leads to the doubling of the periodicity of the dynamic solution. The following Lemma provides conditions for the existence of the Period Doubling bifurcation (see Kuznetsov (1998), p. 415).

Lemma 1 (Period Doubling Bifurcation) Suppose that a one dimensional system

$$
x \mapsto f(x, \alpha), x \in \Re^{1}, \alpha \in \Re^{2},
$$

with $f$ smooth, so that at $\alpha=0$ the fixed point $x=0$, and let the period doubling bifurcation conditions hold:

$$
\mu=f_{x}(0,0)=-1, c=\frac{1}{4}\left[f_{x x}(0.0)\right]^{2}+\frac{1}{6}(0,0)=0 .
$$

Assume, that the following genericity conditions are satisfied:

PD.1 $D(0)=\left(\frac{1}{5} f_{x^{5}}+\frac{1}{2} f_{x} f_{x^{4}}-\left[f_{x}\right]^{4}\right)(0,0)=0 ;$ 
PD.2 the map $\alpha \mapsto(\mu(\alpha)+1, c(\alpha))^{T}$ is regular at $\alpha=0$, where $c(\alpha)$ is given by

$$
c(\alpha)=b(\alpha)+\frac{2 a^{2}(\alpha)}{\mu^{2}(\alpha)-\mu(\alpha)} .
$$

Then there are smooth invertible coordinate and parameter changes transforming the system into

$$
\eta \mapsto-\left(1+\beta_{1}\right) \eta+\beta_{2} \eta^{3}+s \eta^{5}+O\left(\eta^{6}\right), \text { where } s=\operatorname{sign}[D(0)]= \pm 1
$$

This system without $O\left(\eta^{6}\right)$ terms is called the truncated normal form for the period doubling bifurcation.

For our model, both conditions for the period doubling bifurcation hold. To find the combination of parameters that make the variable $\mu$ from Lemma 1 equal to negative one, we analyze the eigenvalues of the characteristic polynomial. The characteristic polynomial $P(\lambda)$ has the following roots:

$$
\lambda_{1,2}=\frac{1}{2}\left[\frac{\alpha_{1} p_{22}+\alpha_{2} p_{11}}{p_{11}+p_{22}-1} \pm \sqrt{D}\right]
$$

where $D$ is the discriminant defined above.

If one of these roots is in the negative part of the unit circle, then there is a possibility of a period doubling bifurcation, given that the nondegeneracy conditions are satisfied.

From the equation for the roots above, we get $\mu=-1$ whenever one of the roots $\lambda_{1,2}=\frac{1}{2}\left[\frac{\alpha_{1} p_{22}+\alpha_{2} p_{11}}{p_{11}+p_{22}-1} \pm \sqrt{\frac{\left(\alpha_{1} p_{22}+\alpha_{2} p_{11}\right)^{2}}{\left(p_{11}+p_{22}-1\right)^{2}}-\frac{4 \alpha_{1} \alpha_{2}}{p_{11}+p_{22}-1}}\right]$ equals -1 . As a result, we 
can rearrange the expression to produce the condition

$$
\sqrt{\left(\alpha_{1} p_{22}+\alpha_{2} p_{11}\right)^{2}-4 \alpha_{1} \alpha_{2}\left(p_{11}+p_{22}-1\right)}=2\left(p_{11}+p_{22}-1\right)+\left(\alpha_{1} p_{22}+\alpha_{2} p_{11}\right)
$$

that needs to hold for a period doubling bifurcation to occur.

Simplifying this expression gives us

$$
p_{11}\left(1+\alpha_{2}\right)+p_{22}\left(1+\alpha_{1}\right)+\alpha_{1} \alpha_{2}=1
$$

This equation can be described as a bifurcation boundary. The bifurcation boundary is a function of the parameters of the dynamical model.

The bifurcation boundary is the key object in our analysis of the dynamical system. The quality of the solution on either side of the boundary is very different. Thus, when the parameters of the dynamical system are close or at the bifuraction boundary, small changes to of parameters can lead to entirely different behavior of the solution. We chose critical bifurcation parameter to be $p_{22}^{c}$.

To calibrate the economy, we use the values in Table 1 . One of the policy regimes, regime 1 , is active with a coefficient greater than 1 whereas regime 2 is a passive regime. The time preference factor $\beta$, reaction of inflation to the output gap $\kappa$, and the degree of relative risk aversion $\sigma$ will only become relevant in the latter part of the paper.

We furthermore assume that the probability of staying in the active regime conditional on being in the active regime $p_{11}=0$ is zero. Whenever regime 1 occurs, the economy will be sent to a passive regime with certainty. 


\begin{tabular}{|l|c|}
\hline Parameter & Value \\
\hline$\alpha_{1}$ & 1.5 \\
$\alpha_{2}$ & 0.5 \\
$\gamma_{1}$ & 0.3 \\
$\gamma_{2}$ & 0.15 \\
$p_{11}$ & 0.85 \\
$p_{22}$ & 0.9 \\
$\beta$ & 0.98 \\
$\kappa$ & 0.024 \\
$\sigma$ & 0.3 \\
\hline
\end{tabular}

Table 1: Standard parameter combination used throughout the paper.

Using these assumptions, we determine the critical value for the transitional probability $p_{22}$ being $p_{22}^{c}=0.1$. We use this point as a benchmark to trace out the bifurcation boundary. To obtain the entire bifurcation boundary, we vary the other parameters, i.e. policy parameters $\alpha_{1}$ and $\alpha_{2}$, along with the probability of staying in the passive regime $p_{22}$.

Consequently, we demonstrate a period doubling bifurcation boundary as a function of the three control parameters $p_{22}, \alpha_{1}$, and $\alpha_{2}$ which is shown in Figure 1. This figure illustrates a bifurcation boundary for the feasible set of parameters where transitional probability $p_{22}$ is between 0 and 1 and parameters reflecting the reaction to inflation are greater than zero.

The graphs shows the shape of the period doubling bifurcation boundary. First, if $p_{22}=1$, then the policy regime would be passive and stay passive indefinitely. In this case, no bifurcation can arise and the bifurcation boundary converges to zero. Second, for the case of $p_{22}=0$, the two policy regimes are identical the likelihood with which they occur. The bifurcation boundary is thus symmetric along policy parameters $\alpha_{1}$ and $\alpha_{2}$. 


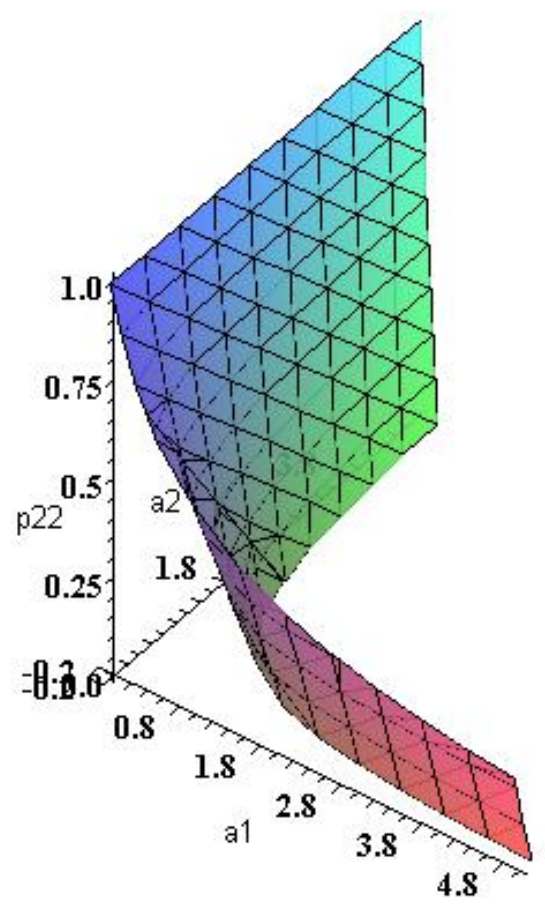

Figure 1: Bifurcation boundary for the case of a simple monetary policy rule keeping the probability $p_{11}=0$.

Interestingly, however, a bifurcation boundary exists for all probabilities between these two extreme cases. In particular, if the policy reaction coefficient $\alpha_{2}$ of the passive regime is small, the policy response coefficient needs be very large for a bifurcation to arise. For a very aggressive policy in the active regime, the rate of inflation will start to evolve in cycles despite the simple nature of its equation of motion. The critical value for this seemingly erratic behavior to occur is plotted in Figure 1. 


\section{New Keynesian model with regime switching}

This section describes the well-known equations for the standard New Keynesian setup as laid out in e.g. Woodford (2003) or Walsh (2003). The standard New Keynesian model traditionally consists of the forward-looking IS equation that describes the demand side of the economy

$$
x_{t}=E_{t} x_{t+1}-\frac{1}{\sigma}\left(i_{t}-E_{t} \pi_{t+1}\right)+u_{t}^{D}
$$

and the Phillips curve which represents the supply side

$$
\pi_{t}=\beta E_{t} \pi_{t+1}+\kappa x_{t}+u_{t}^{S}
$$

The IS curve relates the output gap $x_{t}$ to the nominal interest rate $i_{t}$ and expectations about the future output gap as well as inflation. The coefficient on the difference between the nominal interest rate and expected inflation, i.e. the coefficient on the real interest rate by the Fisher equation, is given by $1 / \sigma$. This coefficient is the inverse of relative risk aversion which equals the elasticity of intertemporal substitution since preferences with constant relative risk aversion are assumed to derive the equations. The New Keynesian Phillips curve describes how inflation is driven by the output gap and expected inflation. Both equations for demand and supply side allow for a shocks $u_{t}$.

The remaining equation to close the economy is a rule for monetary policy which takes the form described in Taylor (1999). According to this Taylor rule, the monetary authority sets the nominal interest rate by targeting both inflation 
and the output gap

$$
i_{t}=\alpha\left(s_{t}\right) \pi_{t}+\gamma\left(s_{t}\right) x_{t}
$$

where $\alpha_{i}$ governs the Central bank's reaction to inflation and $\gamma_{i}$ the reaction to the output gap.

After plugging the Taylor rule into the IS equation, the model can be written in matrix notation ${ }^{1}$

$$
\mathbf{A} \mathbf{Y}_{t+1}=\mathbf{B} \mathbf{Y}_{t}+\mathbf{u}_{t}
$$

where the $\mathbf{Y}$ denotes the vector of variables $\mathbf{Y}=\left[\begin{array}{llll}\pi_{1 t} & \pi_{2 t} & x_{1 t} & x_{2 t}\end{array}\right]^{T}$ and $\mathbf{u}_{t}$ the vector of aggregate demand and supply shocks. The matrix multiplying next period's endogenous variables, inflation and output in either policy regime, is given by

$$
A=\left[\begin{array}{cccc}
\beta p_{11} & \beta\left(1-p_{22}\right) & 0 & 0 \\
\beta\left(1-p_{11}\right) & \beta p_{22} & 0 & 0 \\
\frac{p_{11}}{\sigma} & \frac{1-p_{22}}{\sigma} & p_{11} & 1-p_{22} \\
\frac{1-p_{11}}{\sigma} & \frac{p_{22}}{\sigma} & 1-p_{11} & p_{22}
\end{array}\right] .
$$

The matrix multiplying current period's variables is given by

$$
B=\left[\begin{array}{cccc}
1 & 0 & -\kappa & 0 \\
0 & 1 & 0 & -\kappa \\
\frac{\alpha_{1}}{\sigma} & 0 & 1+\frac{\gamma_{1}}{\sigma} & 0 \\
0 & \frac{\alpha_{2}}{\sigma} & 0 & 1+\frac{\gamma_{2}}{\sigma}
\end{array}\right] .
$$

\footnotetext{
${ }^{1}$ For a detailed derivation of this form see Barnett and Duzhak (2010).
} 
Pre-multiplying both sides of the equation by the inverse of the matrix $\mathbf{A}$, we obtain the normal form

$$
\mathbf{Y}_{t+1}=\mathbf{C} \mathbf{Y}_{t}+\mathbf{A}^{-1} \mathbf{u}_{t}
$$

which we used in the previous section where $\mathbf{C}=\mathbf{A}^{-1} \mathbf{B}$.

After going through the steps of the previous section where we compute the determinant of the matrix $\mathbf{A}$ and then compute the reaction matrix to current period variables, we get

$C=\left[\begin{array}{cccc}\frac{p_{22}}{\left(-1+p_{22}+p_{11}\right) \beta} & \frac{-1+p_{22}}{\left(-1+p_{22}+p_{11}\right) \beta} & -\frac{p_{22} k}{\left(-1+p_{22}+p_{11}\right) \beta} & -\frac{\left(-1+p_{22}\right) k}{\left(-1+p_{22}+p_{11}\right) \beta} \\ \frac{-1+p_{11}}{\left(-1+p_{22}+p_{11}\right) \beta} & \frac{p_{11}}{\left(-1+p_{22}+p_{11}\right) \beta} & -\frac{\left(-1+p_{11}\right) k}{\left(-1+p_{22}+p_{11}\right) \beta} & -\frac{p_{11} k}{\left(-1+p_{22}+p_{11}\right) \beta} \\ \frac{p_{22}\left(-1+\alpha_{1} \beta\right)}{\sigma \beta\left(-1+p_{22}+p_{11}\right)} & \frac{1-p_{22}-\alpha_{2} \beta+\alpha_{2} \beta, p_{22}}{\sigma \beta\left(-1+p_{22}+p_{11}\right)} & \frac{p_{22}\left(k+\sigma \beta+\beta, \gamma_{1}\right)}{\sigma \beta\left(-1+p_{22}+p_{11}\right)} & \frac{\left(-1+p_{22}\right)\left(k+\sigma \beta+\beta \gamma_{2}\right)}{\sigma \beta\left(-1+p_{22}+p_{11}\right)} \\ \frac{1-p_{11}-\alpha_{1} \beta+\alpha_{1} \beta, p_{11}}{\sigma \beta\left(-1+p_{22}+p_{11}\right)} & \frac{p_{11}\left(-1+\alpha_{2} \beta\right)}{\sigma \beta\left(-1+p_{22}+p_{11}\right)} & \frac{\left(-1+p_{11}\right)\left(k+\sigma \beta+\beta \gamma_{1}\right)}{\sigma \beta\left(-1+p_{22}+p_{11}\right)} & \frac{p_{11}\left(k+\sigma, \beta+\beta, \gamma_{2}\right)}{\sigma \beta\left(-1+p_{22}+p_{11}\right)}\end{array}\right]$

As before, matrix $\mathbf{C}$ is the key element when performing bifurcation analysis of the model with a generalized Taylor rule.

Given the standard calibration provided in Table 1, we search the parameter space of the elasticity of inflation with respect to the output gap $\kappa$ and the Taylor coefficient on the output gap in state $2 \gamma_{2}$ for possible bifurcations. In order to locate bifurcation values, we need to choose our free parameter. Parameters that describe the probabilities of regime change are held constant, while structural and policy parameters are varied.

At this stage, however, we need to deviate from the path we took for the basic model of the previous section. While we dealth with a two-by-two matrix in the simple setup, we now have a four-dimensional dynamical system. As a result, 
the computation of the characteristic polynomial and its solution becomes more involved.

Therefore, we move to a numerical methods to study our dynamical system. To perform bifurcation analysis and search for period doubling (PD) and Hopf bifurcations, we employ the software continuation package CONTENT. This dynamical system software, developed by Yuri Kuznetsov and V. V. Levitin, traces out bifurcation boundaries for large dynamical systems.

Due to the increased dimensionality of the system, we have four eigenvalues for the Jacobian matrix where we have two pairs of complex-conjugate eigenvalues. Either pair has the potential to display the bifurcation patterns we discussed previously. Therefore, we could potentially see a Hopf bifurcation or a period doubling (PD) bifurcation but also the combination of the two. Then, for example, the output gap could display a qualitatively different pattern in its evolution from inflation.

When using the software, we can show that none of the possible bifurcations can occur for any feasible set of parameters. Hence there is neither a possibility of a Hopf, nor a possibility of a PD bifurcation, nor the combination thereof for feasible parameters. We do, however, find the bifurcation boundary. A PD-Hopf bifurcation occurs for parameter values $\gamma_{2}=0.179$ and $\kappa=-0.46$. However, since negative values for $\kappa$ are economically infeasible, this is not a relevant case. After tracing out the entire bifurcation boundary, it never crosses into the subspace of feasible parameter combinations. Hence we conclude that given the standard parameterization, the general Taylor rule leads to a structurally stable model. 


\section{The New Keynesian model with a hybrid monetary policy rule}

This section provides an analysis of the state-of-the-art model for monetary

policy. The monetary policy rule consists of a hybrid rule which includes both a current-looking and a forward-looking component. Generally this type of rules can include the features of backward-looking rules such as past values of inflation or output gap, but we will limit our analysis to the following specification

$$
i_{t}=\alpha\left(s_{t}\right) \pi_{t+1}+\gamma\left(s_{t}\right) x_{t} .
$$

This form of a policy rule was proposed by Clarida, Gali and Gertler (1999) where they provide support for a superior to the policy implemented by the Federal Reserve. According to this rule, a policy maker is forward-looking with respect to inflation and current looking with respect to the output gap.

Using this type of monetary policy rule in a New Keynesian setup produces the system linear difference equations

$$
\mathbf{Y}_{t+1}=\mathbf{D} \mathbf{Y}_{t}
$$


where matrix $\mathbf{D}$ is given by

$$
\mathbf{D}=\left[\begin{array}{cccc}
\frac{p_{22}}{\beta\left(-1+p_{22}+p_{11}\right)} & \frac{-1+p_{22}}{\beta\left(-1+p_{22}+p_{11}\right)} & -\frac{p_{22} k}{\beta\left(-1+p_{22}+p_{11}\right)} & -\frac{\left(-1+p_{22}\right) k}{\beta\left(-1+p_{22}+p_{11}\right)} \\
\frac{-1+p_{11}}{\beta\left(-1+p_{22}+p_{11}\right)} & \frac{p_{11}}{\beta\left(-1+p_{22}+p_{11}\right)} & -\frac{\left(-1+p_{11}\right) k}{\beta\left(-1+p_{22}+p_{11}\right)} & -\frac{p_{11} k}{\beta\left(-1+p_{22}+p_{11}\right)} \\
\frac{p_{22}\left(-1+\alpha_{1}\right)}{\sigma \beta\left(-1+p_{22}+p_{11}\right)} & \frac{\left(-1+p_{22}\right)\left(-1+\alpha_{2}\right)}{\sigma \beta\left(-1+p_{22}+p_{11}\right)} & \frac{p_{22}\left(k-k \alpha_{1}+\sigma \beta+\beta \gamma_{1}\right)}{\sigma \beta\left(-1+p_{22}+p_{11}\right)} & \frac{\left(-1+p_{22}\right)\left(k-k \alpha_{2}+\sigma \beta+\beta \gamma_{2}\right)}{\sigma \beta\left(-1+p_{22}+p_{11}\right)} \\
\frac{\left(-1+p_{11}\right)\left(-1+\alpha_{1}\right)}{\sigma \beta\left(-1+p_{22}+p_{11}\right)} & \frac{\left(-1+\alpha_{2}\right) p_{11}}{\sigma \beta\left(-1+p_{22}+p_{11}\right)} & \frac{\left(-1+p_{11}\right)\left(k-k \alpha_{1}+\sigma \beta+\beta \gamma_{1}\right)}{\sigma \beta\left(-1+p_{22}+p_{11}\right)} & \frac{p_{11}\left(k-k \alpha_{2}+\sigma \beta+\beta \gamma_{2}\right)}{\sigma \beta\left(-1+p_{22}+p_{11}\right)}
\end{array}\right] .
$$

We analyze the coefficient matrix $\mathbf{D}$ for possibilities of Hopf and period doubling bifurcations using the same steps as in the preceding section.

Numerical analysis of this dynamic system leads to two findings. First, there is no possibility of a Hopf bifurcation. Second, however, a period doubling bifurcation emerges. The findings are thus the same as for the simple economy.

Figure 2 plots the bifurcation boundary for the period-doubling bifurcation for the standard parameter combination of 1 where we allow risk aversion and the policy response $\alpha_{2}$ to vary.

To find a bifurcation boundary we need to choose a parameter that will be varied. Assume parameter $\alpha_{2}$ is a free parameter that we use for a numerical bifurcation analysis. We first vary parameter $\alpha_{2}$ while holding all other parameters constant. Assuming the standard calibration in Table 1, the critical value of parameter $\alpha_{2}$ is 0.00125 . We use this point to trace out the bifurcation boundary. After tracking the first period doubling bifurcation point, we chose the second parameter that is varied simultaneously with parameter $\alpha_{2}$. For that case, we can demonstrate a period doubling bifurcation boundary as a function of two control parameters, $\alpha_{2}$ and the risk aversion parameter $\sigma$. Figure 2 shows 


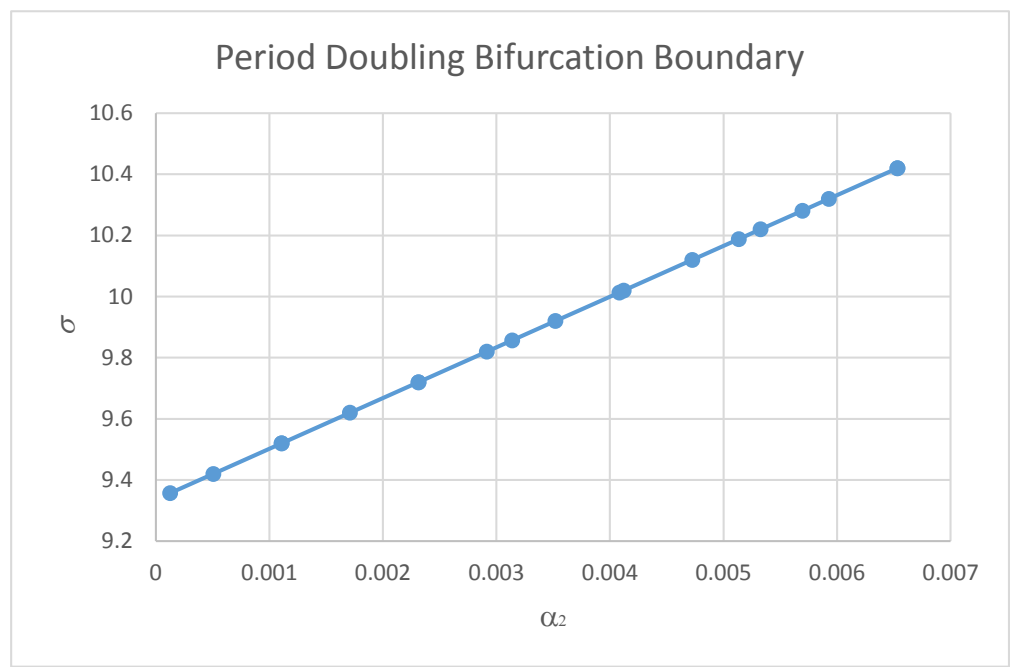

Figure 2: Bifurcation for the period-doubling bifurcation in a model with a hybrid policy rule where we vary parameters $\alpha_{2}$ and $\sigma$.

this period doubling bifurcation boundary which has values of parameter $\alpha_{2}$ in the close proximity of zero. Hence, a period doubling bifurcation will occur for a very narrow set of parameters $\alpha_{2}$ corresponding to a passive reaction to future inflation. Similarly, we find a period doubling bifurcation point for parameter $\kappa=3.725$. After choosing a second parameter, $\sigma$, to be varied, we compute the period doubling bifurcation boundary demonstrated by Figure 3. Parameter $\kappa$ is a nonlinear function of the discount factor and the parameter responsible for the degree of price rigidity. It shows that the period doubling bifurcation will occur when the economy is characterized by a high level of price stickiness.

After analyzing further parameter combinations, we find that a period doubling bifurcation is also possible for lower values of $\kappa$ accompanied by very high values of the policy parameter $\alpha_{1}$ as shown in Figure 4. In other words, aggressive 


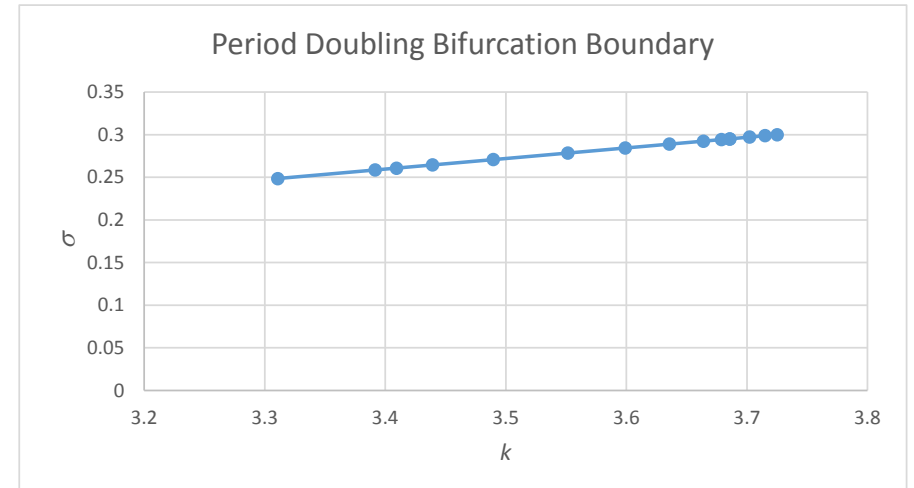

Figure 3: Bifurcation for the period-doubling bifurcation in a model with a hybrid policy rule where we vary parameters $\sigma$ and $\kappa$.

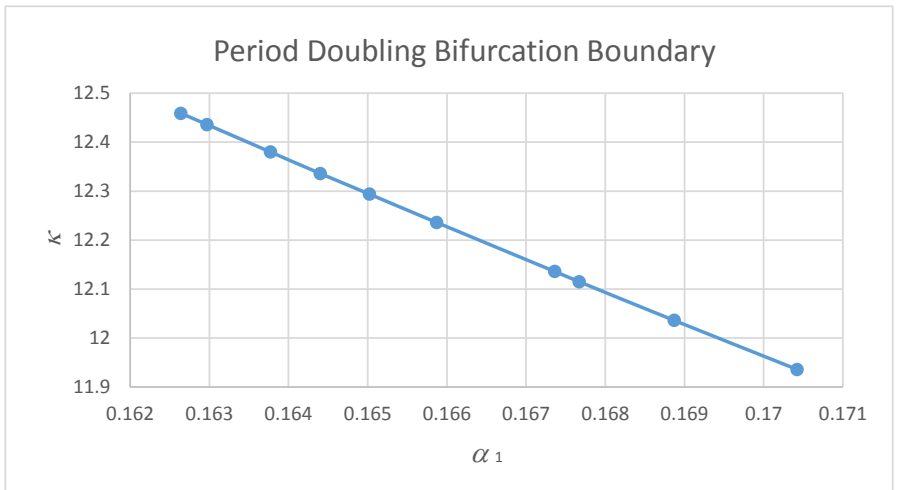

Figure 4: Bifurcation for the period-doubling bifurcation in a model with a hybrid policy rule where we vary parameters $\alpha_{1}$ and $\kappa$. 
reaction of the Central bank to future inflation will lead to a period doubling bifurcation.

\section{Conclusion}

The New Keynesian model with a Taylor rule generalized to regime switching allows policy parameters to vary over time. This type of policy rule can change the dynamics of inflation and the output gap in a substantial way. We show that not only short-term properties change but that regime switching can give rise to changes in the qualitative properties of the solution. To get to this conclusion, we analyze the dynamical properties of these models via bifurcation analysis. We look for Hopf and period doubling bifurcations within the functional structure considered. The analytical bifurcation analysis detects the possibility of a period doubling bifurcation for a simple monetary rule using Fisher's equation. This result carries over to the state-of-the-art monetary model with a hybrid Taylor rule which is shown to be subject to a period doubling bifurcation. Our analysis reveals that period doubling bifurcations and resulting changes in the dynamics in inflation and output have more tendencies to arise in New Keynesian model with the forward-looking generalized Taylor rule compared to the current-looking counterpart. 


\section{References}

Andrews, Donald W. K., "Tests for Parameter Instability and Structural Change with Unknown Change Point," Econometrica, 1993, 61 (4), 821-856.

Barnett, William A. and Evgeniya A. Duzhak, "Non-robust Dynamic Inferences from Macroeconometric Models: Bifurcation Stratification of Confidence Regions," Physica A, 2008, 387 (15), 3817-3825.

_ and _ , "Empirical Assessment of Bifurcation Regions within New Keynesian Models," Economic Theory, 2010, 45 (1-2), 99-128.

_ and Yijun He, "Stability Analysis of Continuous-Time Macroeconometric Systems," Studies in Nonlinear Dynamics and Econometrics, 1999, 3 (4), $169-188$.

_ and _, "Nonlinearity, Chaos, and Bifurcation: A Competition and an Experiment," in Negishi, Ramachandran, and Kazuo Mino (eds.), Economic Theory, Dynamics and Markets: Essays in Honor of Ryuzo Sato, Kluwer Academic Publishers, 2001, pp. 167-187.

_ and _ , "Stabilization Policy as Bifurcation Selection: Would Stabilization Policy Work if the Economy Really Were Unstable?," Macroeconomic Dynamics, 2002, 6 (5), 713-747.

_ and _ , "Bifurcations in Macroeconomic Models," in Steve Dowrick, Rohan Pitchford, and Steven Turnovsky (eds), Economic Growth and Macroeconomic Dynamics: Recent Developments in Economic Theory, Cambridge University Press, 2004, pp. 95-112. 
_ and _, "Robustness of Inferences to Singularity Bifurcation," Proceedings of the Joint Statistical Meetings of the 2005 American Statistical Society, American Statistical Society, 2006, 100.

Benhabib, Jess and Kazuo Nishimura, "The Hopf Bifurcation and the Existence and Stability of Closed Orbits in Multisector Models of Optimal Economic Growth," Journal of Economic Theory, 1979, 21 (3), 421 â444.

Bernanke, Ben S., Thomas Laubach, Frederic S. Mishkin, and Adam S. Posen, Inflation Targeting: Lessons from the International Experience, Princeton, NJ: Princeton University Press, 1999.

Clarida, Richard, Jordi Gali, and Mark Gertler, "The Science of Monetary Policy: A New Keynesian Perspective," Journal of Economic Literature, 1999, 37 (Dec), 1661â1707.

Davig, Troy and Eric M. Leeper, "Generalizing the Taylor Principle," American Economic Review, 2006, 97 (3), 607-635.

Evans, George, "Expectational Stability and the Multiple Equilibria Problem in Linear Rational Expectations Models," Quarterly Journal of Economics, 1985, 100 (4), 1217-1233.

Farmer, Roger E. A., Daniel F. Waggoner, and Tao Zha, "Understanding the New Keynesian Model When Monetary Policy Switches Regimes," Working Paper 2007-12, NBER Working Paper No. 129652007.

Gali, Jordi and Mark Gertler, "Inflation dynamics: A Structural Econometric Analysis," Journal of Monetary Economics, 1999, 44 (2), 195-222. 
Groen, Jan J. J. and Haroon Mumtaz, "Investigating the Structural Stability of the Phillips Curve Relationship," Bank of England Working Paper No. 350, 2008.

Hamilton, James D., "A New Approach to the Economic Analysis of Nonstationary Time Series and the Business Cycle," Econometrica, 1989, 57, $357-384$.

Hansen, Bruce E., "Testing for Parameter Instability in Linear Models," Journal of Policy Modeling, 1992, 14 (4), 517â533.

Hopf, Eberhard, "Abzweigung Einer Periodischen Lösung von Einer Stationaren Lösung Eines Differentialsystems," Sáchsische Akademie der Wissenschaften Mathematische-Physikalische, Leipzig, 1942, 94 (1-22).

Kuznetsov, Yuri A., Elements of Applied Bifurcation Theory, SpringerVerlag, New York, 1998.

Leeper, Edward and Christopher A. Sims, "Toward a Modern Macro Model Usable for Policy Analysis," NBER Macroeconomics Annual, 1994, (81-117).

Nyblom, Jukka, "Testing for the Constancy of Parameters over Time," Journal of the American Statistical Association, 1989, 84 (405).

Seydel, Rüdiger, Practical Bifurcation and Stability Analysis, SpringerVerlag, New York, 1994.

Sims, Christopher A. and Tao Zha, "Were There Regime Switches in U.S. Monetary Policy?," American Economic Review, 2006, pp. 54-81. 
Taylor, John B., "A Historical Analysis of Monetary Policy Rules," in John B. Taylor, ed., Monetary Policy Rules, Chicago: University of Chicago Press for NBER, 1999, pp. 319-40.

Walsh, Carl E., Monetary Theory and Policy, second ed., MIT Press: Cambridge Mass, 2003.

Warne, Anders, "Causality and Regime Inference in a Markov Switching VAR," Sveriges Riksbank Working Paper No. 118, 2000.

Woodford, Michael, Interest and Prices: Foundations of a Theory of Monetary Policy, Princeton, NJ: Princeton University Press, 2003. 